\title{
BUDDY-system: A web site for constructing a dataset of protein pairs between ligand-bound and unbound states
}

Mizuki Morita ${ }^{1,2^{*}}$, Tohru Terada ${ }^{3}$, Shugo Nakamura ${ }^{4}$ and Kentaro Shimizu ${ }^{2,4}$

\begin{abstract}
Background: Elucidating molecular recognition by proteins, such as in enzyme-substrate and receptor-ligand interactions, is a key to understanding biological phenomena. To delineate these protein interactions, it is important to perform structural bioinformatics studies relevant to molecular recognition. Such studies require a dataset of protein structure pairs between ligand-bound and unbound states. In many studies, the same welldesigned and high-quality dataset has been used repeatedly, which has spurred the development of subsequent relevant research. Using previously constructed datasets, researchers are able to fairly compare obtained results with those of other studies; in addition, much effort and time is saved. Therefore, it is important to construct a refined dataset that will appeal to many researchers. However, constructing such datasets is not a trivial task.

Findings: We have developed the BUDDY-system, a web site designed to support the building of a dataset comprising pairs of protein structures between ligand-bound and unbound states, which are widely used in various areas associated with molecular recognition. In addition to constructing a dataset, the BUDDY-system also allows the user to search for ligand-bound protein structures by its unbound state or by its ligand; and to search for ligands by a particular receptor protein.

Conclusions: The BUDDY-system receives input from the user as a single entry or a dataset consisting of a list of ligand-bound state protein structures, unbound state protein structures, or ligands and returns to the user a list of protein structure pairs between the ligand-bound and the corresponding unbound states. This web site is designed for researchers who are involved not only in structural bioinformatics but also in experimental studies. The BUDDY-system is freely available on the web.
\end{abstract}

\section{Findings}

Elucidating molecular recognition by proteins is one of the keys to understanding biological phenomena. Structural bioinformatics studies relevant to molecular recognition, such as analysis of conformational changes upon ligand binding [1-4], development of methods for predicting ligand binding sites [5-7], and development of molecular docking tools [8-10], require a dataset of protein structure pairs between ligand-bound and unbound states (Figure 1).

The BUDDY-system features a flexible definition of a ligand and allows the user to change various options via

\footnotetext{
* Correspondence: mizuki@bi.a.u-tokyo.ac.jp

'Department of Fundamental Research, National Institute of Biomedical

Innovation (NIBIO), 7-6-8 Saito Asagi, Ibaraki, Osaka 567-0085, Japan

Full list of author information is available at the end of the article
}

its web interface. The BUDDY-system is based on a premise that differs from existing structural bioinformatics systems in terms of what is considered a ligand. In previous studies, a ligand was defined as all heterogeneous (HETATM) molecules in the Protein Data Bank (PDB) [11] files [1,12], all HETATM molecules except for lowmolecular ions (e.g., $\mathrm{Zn}^{2+}, \mathrm{Mn}^{2+}, \mathrm{PO}_{4}{ }^{3-}$, and $\mathrm{SO}_{4}{ }^{2-}$ ) [4], or HETATM clusters forming many inter-atomic contacts with protein atoms [13]. This variety of ligand definitions implies that it is very difficult to specifically define a ligand. Here, we define a ligand as molecules that can dissociate from proteins; consequently, a certain protein can be found with a ligand in some entries in PDB and without it in other entries. Under this definition, a ligand is not determined specifically but instead depends on each pair of PDB entries. For example, the

\section{Biomed Central}




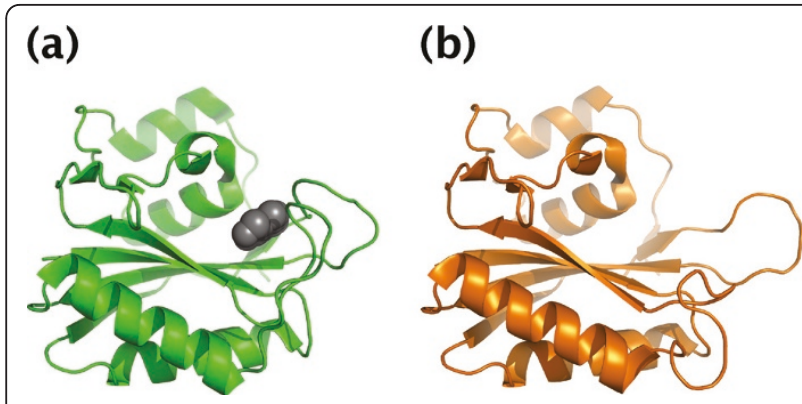

Figure 1 Example of a protein structure pair between ligandbound and unbound states. (a) A ligand-bound state structure of 6-hydroxymethyl-7,8-dihydropterin pyrophosphokinase (HPPK) ([PDB:1HQ2]) and (b) an unbound state structure ([PDB:1 IM6]). The ligand is represented by dark spheres. The BUDDY-system allows users to search for this type of pair by its ligand as a search query, bound states and ligands by its unbound state, and unbound states from its bound state. The user can input 1 query or a set of such queries as a dataset.

structure of fructose-1,6-bisphosphatase (F16BPase) [14], which catalyzes the hydrolysis of d-fructose 1,6-bisphosphate (FBP) to d-fructose 6-phosphate (F6P) and phosphate $(\mathrm{Pi})$, has been demonstrated several times in different binding states (Figure 2): F16BPase in free form ([PDB:2FBP]); with F6P in the active site ([PDB:1RDX]); with F6P and adenosine monophosphate (AMP) in the allosteric site ([PDB:1FBP]); and with F6P, $\mathrm{AMP}$, and the anilinoquinazoline inhibitor (PFE) in the non-native allosteric site ([PDB:1KZ8]). If a ligand is defined specifically as "HETATM molecules except for low-molecular ions," [PDB:2FBP] would be reported as existing in the ligand-unbound state and all the others in the ligand-bound state. However, although [PDB:1RDX] exists in the ligand-bound state against [PDB:2FBP], it also exists in the ligand-unbound state against [PDB:1FBP] and [PDB:1KZ8]. Likewise, while [PDB:1FBP] is in the ligand-bound state against [PDB:2FBP] and [PDB:1RDX], it is also present in a ligand-unbound state against [PDB:1KZ8]. The flexible ligand definition in the BUDDY-system enables the user to obtain all possible ligand-bound and unbound state pairs of F16BPase.

We plan to implement more advanced search options in the future, such as protein sequence similarity search and chemical structure search from SMILES.

\section{Methods}

The procedure for constructing a dataset of protein pairs between ligand-bound and unbound states (called bound/unbound-pairs) in the BUDDY-system consists of the following 3 steps: (1) finding all pairs of the same proteins or homologues in all the PDB entries to prepare an initial dataset, (2) screening bound/unbound-

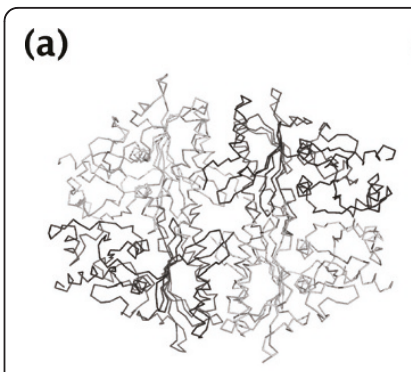

(b)

(c)

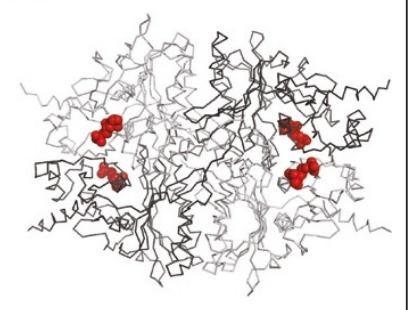

(d)
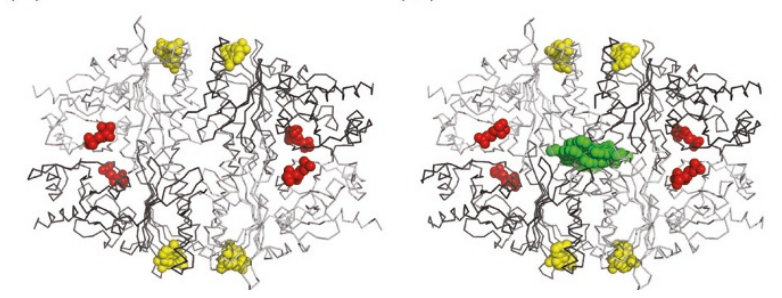

Figure 2 Examples illustrating the difficulty in defining a ligand. F16BPase tetramer (a) in free form ([PDB:2FBP]), (b) with F6P (red sphere) in the active site ([PDB:1RDX]), (c) with F6P and AMP (yellow sphere) in the allosteric site ([PDB:1FBP]), and (d) with F6P, $A M P$, and PFE (green sphere) in the non-native allosteric site ([PDB:1KZ8]). Although [PDB:1RDX] is in a ligand-bound state against [PDB:2FBP], it is also in a ligand-unbound states against [PDB:1FBP]. Further, although [PDB:1FBP] is in a ligand-bound state against [PDB:2FBP] and [PDB:1RDX], it is also in a ligand-unbound state against [PDB:1KZ8]. If a ligand is defined specifically as "HETATM molecules except for low-molecular ions," all entries but [PDB:2FBP] are obtained in a ligand-bound state. The flexible ligand definition in the BUDDY-system enables the user to obtain all possible ligandbound and unbound states pairs of F16BPase.

pairs from the initial dataset to prepare a super dataset, and (3) finding suitable pairs for the user's request from the super dataset after the user submits a request (Figure 3). The first 2 steps are carried out in advance, and the third step can be achieved after the user enters input data. The details are as follows. (1) The BUDDYsystem finds pairs of the same proteins or homologues from all of the PDB entries based on their sequence identity to prepare an initial dataset (the sequence identity threshold can be specified by the user via the web interface). Here, a chain shorter than $N$ amino acids is defined as "a peptide chain" and is considered a ligand ( $N$ can be specified by the user via the web interface). This option is useful, especially when a protein has short amino acid chains that are essential for its function (e.g., insulin). (2) Next, the BUDDY-system screens the bound/unbound-pairs to prepare a super dataset from the initial dataset. Initially, the BUDDY-system compiles HETATM lists of both PDB entries in a pair, respectively. Here, when an HETATM molecule appears more than once in a PDB entry, it is listed only once in its HETATM list. Furthermore, HETATM molecules 


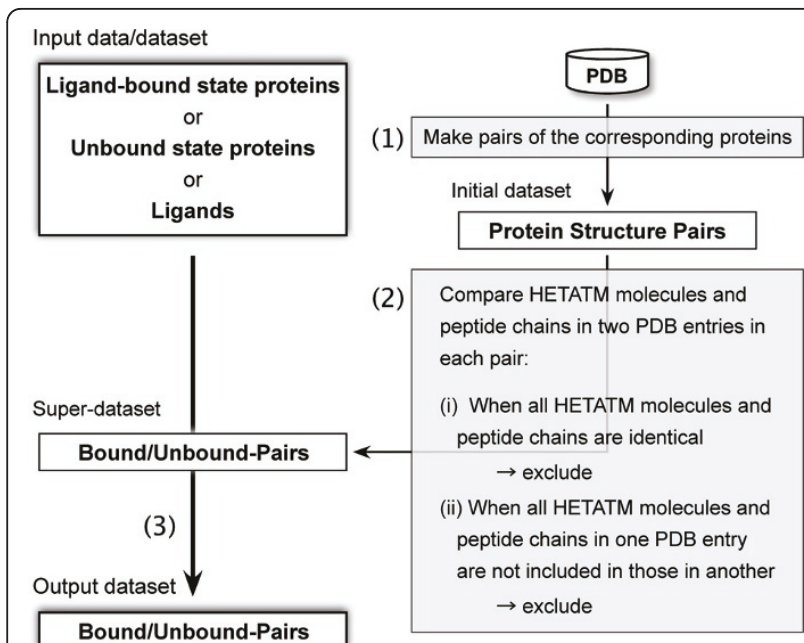

Figure 3 Schematic diagram illustrating the construction of a dataset in the BUDDY-system. The process of constructing a pair dataset consists of the following 3 steps: (1) all pairs of the same proteins or homologues are obtained from the entire PDB entries to prepare an initial dataset, (2) protein structure pairs of ligand-bound and unbound states are screened from the initial dataset to prepare a super dataset, and (3) the pairs that fit the user's request are selected from the super dataset after the user submits a request.

that are defined as "not considered as a ligand" will be excluded from the HETATM list. If the PDB file has chains shorter than $N$ amino acids in the ATOM record ( $N$ can be decided by the user via the web interface), they are considered "peptide chains." The BUDDY-system then compares 2 HETATM lists and peptide chains from 2 PDB entries in a pair and judges whether this pair is a bound/unbound-pair in the following manner: (2-i) when the contents of 2 HETATM lists and peptide chains are identical, this pair is not regarded as a bound/unbound-pair; and (2-ii) when those are not identical, a pair is a bound/unbound-pair if 1 HETATM list is included in another list. (3) Finally, after the user inputs ligand-bound state protein structures, unbound state protein structures, or ligands into the BUDDY-system, bound/unbound-pairs that fit the user's request are selected from the super dataset and are returned to the user. The user can (3-i) upload their own datasets including a PDB ID list of ligand-bound state protein structures, unbound state protein structures, or a HETATM ID list of ligands; (3-ii) choose one of the readymade datasets of ligand-bound state protein structures, such as BindingDB [15] and PLD [16] (whose use of each has been generously permitted by the authors listed in references 14 and 15, respectively); or (3-iii) input one PDB ID of a ligand-bound state protein structure or an unbound state protein structure, or one HETATM ID of a ligand. The file formats of the input and output datasets are described on the BUDDY- system website. The parameters that the user can select are the cut-off value of $\mathrm{X}$-ray resolution, the sequence identity when making a bound/unbound-pair, and the definition of a peptide chain.

\section{Example Usage}

Here, we show examples of using the BUDDY-system. Table 1 shows the results obtained from the BUDDYsystem when a list of PDB entries of ligand-bound state proteins, which were obtained from various databases or datasets available on the Internet, were input with the following default parameters: X-ray resolutions equal to or better than $2.5 \AA$ were allowed, a sequence identity between ligand-bound and unbound state protein equal to $100 \%$ was used, and chains shorter than 30 amino acids were considered peptide ligands. In the example shown in Table 1, when PDB entries obtained from BindingDB were input, at least 1 corresponding unbound state entry was found for 484 of 1,485 input ligandbound state protein entries, and the number of total pairs was 4,629. Interestingly, at least 1 unbound state PDB entry was found for approximately $30 \%$ of the input ligand-bound state protein structures for any of the datasets in Table 1. Additionally, a large portion of these ligand-bound state structures was paired with only 1 corresponding unbound state protein structure. Although this number of returned pairs would increase or decrease depending on the parameters used, these examples in Table 1 demonstrate that a dataset of

Table 1 Summary of the results obtained using the BUDDY-system against various datasets

\begin{tabular}{|c|c|c|c|c|}
\hline \multirow[t]{2}{*}{ Input dataset $^{a}$} & \multirow[t]{2}{*}{ Version $^{\mathbf{b}}$} & \multicolumn{3}{|c|}{$\begin{array}{l}\text { The number of protein } \\
\text { structures }\end{array}$} \\
\hline & & Input $^{c}$ & Output $A^{d}$ & Output $\mathrm{B}^{\mathrm{e}}$ \\
\hline AffinDB [17] & 2008-03-17 & 476 & 157 & 2,257 \\
\hline BindingDB [15] & 2008-03-17 & 1,485 & 484 & 4,629 \\
\hline PDBbind [18] & v2007 & 3,124 & 996 & 7,000 \\
\hline PLD [16] & v1.3 & 485 & 137 & 1,277 \\
\hline CCDC/Astex Test Set [8] & - & 305 & 91 & 611 \\
\hline Astex Diverse Set [10] & - & 85 & 25 & 170 \\
\hline Non-redundant ${ }^{f}$ & - & 4,544 & 1,172 & 7,901 \\
\hline
\end{tabular}

This table shows the results obtained from the BUDDY-system when various databases were input as lists of ligand-bound states proteins. Taking BindingDB as an example, when 1,485 PDB entries obtained from BindingDB were input as a ligand-bound state, at least 1 corresponding unbound state PDB entry was found for 484 of 1,485 input entries; a total of 4,629 pairs were obtained.

${ }^{\mathrm{a}}$ Each input dataset contains a list of PDB entries of ligand-bound state proteins.

${ }^{\mathrm{b}}$ The version of the input dataset or the date when the input dataset was downloaded.

'The number of ligand-bound state protein structures in each input dataset.

${ }^{d}$ The number of unique proteins that were paired with at least one ligand-

unbound state entry by the BUDDY-system.

${ }^{\mathrm{e}}$ The number of total pairs.

fUnion of all ligand-bound state protein datasets. 
bound/unbound-pairs can be readily obtained with the BUDDY-system. The datasets obtained here are essential for elucidating molecular recognition by proteins in studies that investigate conformational changes involved in enzyme reactions, developments of ligand binding site prediction, and components involved in molecular docking. The BUDDY-system is the first web site that the authors are aware of that supports the construction of such a dataset according to the user's input dataset and parameters. In addition, because the ligand is allowed a more flexible definition, this web server is useful to exhaustively search for ligands or ligand-bound and unbound state structures that are of interest to the user.

\section{Availability and Requirements}

The BUDDY-system is freely available at URL http:// www.bi.a.u-tokyo.ac.jp/services/buddy/.

\section{Abbreviations}

AMP: Adenosine monophosphate; F16BPase: Fructose-1,6-bisphosphatase; F6P: d-fructose 6-phosphate; HPPK: 6-Hydroxymethyl-7,8-dihydropterin pyrophosphokinase; PDB: Protein Data Bank; Pi: Phosphate.

\section{Acknowledgements}

The authors thank Dr. John Mitchell and Dr. Michael Gilson for granting permission to use their datasets. The authors also thank Dr. Kazuya Sumikoshi for technical contributions. This work was partially supported by Grant-in-Aid for Young Scientists (B) and Grant-in-Aid for Scientific Research on Priority Areas Systems Genomics from the Ministry of Education, Culture, Sports, Science and Technology of Japan.

\section{Author details}

${ }^{1}$ Department of Fundamental Research, National Institute of Biomedical Innovation (NIBIO), 7-6-8 Saito Asagi, Ibaraki, Osaka 567-0085, Japan. ${ }^{2}$ Institute for Bioinformatics Research and Development (BIRD), Japan Science and Technology Agency (JST), 4-1-8 Honcho, Kawaguchi, Saitama 332-0012, Japan. ${ }^{3}$ Agricultural Bioinformatics Research Unit, The University of Tokyo, 1-1-1 Yayoi, Bunkyo, Tokyo 113-8657, Japan. ${ }^{4}$ Department of Biotechnology, The University of Tokyo, 1-1-1 Yayoi, Bunkyo, Tokyo 113-8657, Japan.

\section{Authors' contributions}

MM developed the concept and designed the algorithm and its implementation. TT provided valuable suggestions on the manuscript. SN contributed to the implementation of the web site. KS reviewed and tested the software. All authors read and approved the final version of the manuscript.

\section{Competing interests}

The authors declare that they have no competing interests.

Received: 21 December 2010 Accepted: 22 May 2011 Published: 22 May 2011

\section{References}

1. Najmanovich R, Kuttner J, Sobolev V, Edelman M: Side-Chain Flexibility in Proteins Upon Ligand Binding. Proteins 2000, 39:261-268.

2. Carlson HA: Protein flexibility and drug design: how to hit a moving target. Curr Opin Chem Biol 2002, 6:447-452.

3. Gutteridge A, Thornton J: Conformational Changes Observed in Enzyme Crystal Structures upon Substrate Binding. J Mol Biol 2005, 346:21-28.

4. Gunasekaran K, Nussinov R: How Different are Structurally Flexible and Rigid Binding Sites? Sequence and Structural Features Discriminating
Proteins that Do and Do not Undergo Conformational Change upon Ligand Binding. J Mol Biol 2007, 365:257-273.

5. Brady GP, Stouten PFW: Fast prediction and visualization of protein binding pockets with PASS. J Computer-Aided Mol Design 2000, 14:383-401.

6. Laurie ATR, Jackson RM: Q-SiteFinder: an energy-based method for the prediction of protein-ligand binding sites. Bioinformatics 2005, 21:1908-1916.

7. Morita M, Nakamura S, Shimizu K: Highly accurate method for ligandbinding site prediction in unbound state (apo) protein structures. Proteins 2008, 73:468-479.

8. Nissink JWM, Murray C, Hartshorn M, Verdonk ML, Cole JC, Taylor R: A New Test Set for Validating Predictions of Protein-Ligand Interaction. Proteins 2002, 49:457-471.

9. Meiler J, Baker D: ROSETTALIGAND: Protein-Small Molecule Docking with Full Side-Chain Flexibility. Proteins 2006, 65:538-548.

10. Hartshorn MJ, Verdonk ML, Chessari G, Brewerton SC, Mooij WTM, Mortenson PN, Murray CW: Diverse, High-Quality Test Set for the Validation of Protein-Ligand Docking Performance. J Med Chem 2007, 50:726-741.

11. Berman HM, Westbrook J, Feng Z, Gilliland G, Bhat TN, Weissig H, Shindyalov IN, Bourne PE: The Protein Data Bank. Nucleic Acids Res 2000, 28:235-242.

12. Shin JM, Cho DH: PDB-Ligand: a ligand database based on PDB for the automated and customized classification of ligand-binding structures. Nucleic Acids Res 2005, 33:D238-D241.

13. Dessailly BH, Lensink MF, Orengo CA, Wodak SJ: LigASite-a database of biologically relevant binding sites in proteins with known apostructures. Nucleic Acids Res 2008, 36:D667-D673.

14. Ke HM, Zhang YP, Lipscomb WN: Crystal structure of fructose-1,6bisphosphatase complexed with fructose 6-phosphate, AMP, and magnesium. Proc Natl Acad Sci USA 1990, 87:5243-5247.

15. Liu T, Lin Y, Wen X, Jorissen RN, Gilson MK: BindingDB: a web-accessible database of experimentally determined protein-ligand binding affinities. Nucleic Acids Res 2007, 35:D198-D201.

16. Puvanendrampillai D, Mitchell JBO: Protein Ligand Database (PLD): additional understanding of the nature and specificity of protein-ligand complexes. Bioinformatics 2003, 19:1856-1857.

17. Block P, Sotriffer CA, Dramburg I, Klebe G: AffinDB: a freely accessible database of affinities for protein-ligand complexes from the PDB. Nucleic Acids Res 2006, 34:D522-D526.

18. Wang R, Fang X, Lu Y, Wang S: The PDBbind Database: Collection of Binding Affinities for Protein-Ligand Complexes with Known ThreeDimensional Structures. J Med Chem 2004, 47:2977-2980.

doi:10.1186/1756-0500-4-143

Cite this article as: Morita et al.: BUDDY-system: A web site for constructing a dataset of protein pairs between ligand-bound and unbound states. BMC Research Notes 2011 4:143.

\section{Submit your next manuscript to BioMed Central and take full advantage of:}

- Convenient online submission

- Thorough peer review

- No space constraints or color figure charges

- Immediate publication on acceptance

- Inclusion in PubMed, CAS, Scopus and Google Scholar

- Research which is freely available for redistribution 